\title{
Chapter 1 \\ Peter Marwedel and the Department of Computer Science of the TU Dortmund University
}

\author{
Gernot Fink and Heinrich Müller
}

\subsection{Introduction}

Peter Marwedel was appointed Professor at the Department of Computer Science of the University of Dortmund in 1989. He represented the area "Computer Engineering and Embedded Systems" and headed the Chair of Computer Science 12 until his retirement in 2014. During this time, he has made a great contribution to the Department of Computer Science, which continues to have a lasting effect today. The following presentation is intended to give an idea of the extraordinary breadth and importance of his activities in teaching, academic self-government, basic research, and technology transfer.

\subsection{Teaching}

A special passion of Professor Marwedel is teaching, which is not self-evident for a dedicated and successful researcher. In Dortmund, he was extremely involved in basic teaching, which is particularly challenging given the high number of students attending these courses. For many years he has held the introductory compulsory lecture "Computer Structures" and the elective lectures "Embedded Systems" and "Computer Architecture." Through these courses, he has substantially contributed to the basic training in technical computer science. His lectures were extremely well prepared. The material was thoughtfully selected in all details, didactically carefully prepared, and presented objectively and clearly. The courses have gained a high

\footnotetext{
G. Fink $\cdot$ H. Müller $(\bowtie)$

TU Dortmund, Dortmund, Germany

e-mail: Gernot.Fink@tu-dortmund.de; heinrich.mueller@cs.uni-dortmund.de
} 
reputation among the students. This was reflected in consistently excellent student ratings and led to Professor Marwedel receiving the prestigious Teaching Award of TU Dortmund University in 2003.

From the course "Embedded Systems" the first edition of the English textbook "Embedded System Design" emerged in 2003. The book has established itself as an international textbook and is often cited. Professor Marwedel has adapted it over the years to current developments. The third edition has been published in 2018.

In addition to traditional teaching, Professor Marwedel has shown great interest in the possibilities of new media and has made strong use of it. On YouTube he has made available a significant number of educational videos that are particularly associated with his courses "computer structures" and "computer architecture." The design of the videos focuses on the essentials and avoids superfluous, distracting visual effects.

In his last active years at the department, Professor Marwedel experimented with the concept of the inverted classroom as a natural consequence. This form of teaching is still little used at German universities.

Particularly noteworthy is the fact that Professor Marwedel has conveyed his concept of education in the field of embedded systems beyond its actual implementation. It was the subject of the Workshop on Embedded and Cyber-Physical System Education (WESE) organized by him in Finland in 2012. In the same year, he was invited to the annual meeting of the cyber-physical systems program of the prestigious National Science Foundation (NSF) in Washington, USA, where he gave a talk focusing on the Dortmund education concept in the field of Embedded Systems.

Finally, it should be mentioned that Professor Marwedel contributed to the internationalized teaching of the Department by participating in the English Master's program "Automation and Robotics" of the TU Dortmund University and his contacts to Indian Institutes of Technology, which have led to internships of Indian students in Dortmund.

\subsection{Academic Self-Government}

Professor Marwedel has always been active in the Department's academic administration and beyond. He was Dean of Studies of the Department and member of the Academic Senate of TU Dortmund. But here, too, his commitment to teaching is particularly reflected. He was chairman of the teaching committee of the Department and chairman of the educational committee of the Academic Senate of the university. He was Dean of Studies of the Department from 2012 to 2014. Beyond the university, he has worked on standards for the accreditation of computer science curricula in the national organizations ASIIN and AVI. 


\subsection{Basic Research and SFB 876}

Professor Marwedel has contributed significantly to the international visibility of the Department of Computer Science through his research in embedded and cyberphysical systems. He has received several international honors, in particular the EDAA Lifetime Achievement Award (2013), the ESWEEK Lifetime Achievement Award (2014), and the ACM SIGDA Distinguished Service Award (2014). In 2010 he became an IEEE Fellow and a Fellow of the Design, Automation and Test Conference in Europe (DATE).

A very special contribution to research at the Department and the university was made by Professor Marwedel as co-initiator of the Collaborative Research Center (CRC/SFB) 876, together with the main initiator Prof. Dr. Katharina Morik, spokeswoman for the CRC. The CRC program of the Deutsche Forschungsgemeinschaft (DFG) enjoys a high reputation and is extremely competitive. The topic of the SFB 876 is "Providing Information by Resource-Constrained Data Analysis." The collaborative research center SFB 876 brings together data mining and embedded systems. On the one hand, embedded systems can be improved using machine learning. On the other hand, data mining algorithms can be realized in hardware, e.g. FPGAs, or run on GPGPUs. The restrictions of ubiquitous systems in computing power, memory, and energy demand new algorithms for known learning tasks. At the time of the application, merging data analysis and resource constraints was visionary-today it is highly relevant in many applications. In the SFB 876, about 20 research groups of the Department, of TU Dortmund University, and of neighboring universities are working together since 2011 for 12 years in an interdisciplinary manner.

\subsection{Technology Transfer and ICD}

Professor Marwedel has not only been active in basic research, but has also dealt with applied research and the transfer of scientific results to the economy. For many years he is CEO of the "Informatik Centrum Dortmund e.V." (ICD) and head of the Embedded Systems Group at ICD. The ICD was founded in 1989 from the Department of Computer Science at the University of Dortmund. The ICD is available to companies from all sectors of the economy. Its goal is to accelerate the transfer of current research results in computer science and information technology into industrial products. Under Professor Marwedel's leadership, the ICD has become a well-established, successful, and economically stable association.

\subsection{Conclusion}

The Department of Computer Science is extremely grateful to Professor Marwedel. On the occasion of his seventieth birthday, it wishes him all the best for the future. 
Open Access This chapter is licensed under the terms of the Creative Commons Attribution 4.0 International License (http://creativecommons.org/licenses/by/4.0/), which permits use, sharing, adaptation, distribution and reproduction in any medium or format, as long as you give appropriate credit to the original author(s) and the source, provide a link to the Creative Commons license and indicate if changes were made.

The images or other third party material in this chapter are included in the chapter's Creative Commons license, unless indicated otherwise in a credit line to the material. If material is not included in the chapter's Creative Commons license and your intended use is not permitted by statutory regulation or exceeds the permitted use, you will need to obtain permission directly from the copyright holder. 